\title{
Magnetism in All-Carbon Nanostructures with Negative Gaussian Curvature
}

\author{
Noejung Park, ${ }^{1,2}$ Mina Yoon, ${ }^{3}$ Savas Berber, ${ }^{3}$ Jisoon Ihm, ${ }^{4,2}$ Eiji Osawa, ${ }^{5}$ and David Tománek ${ }^{3, *}$ \\ ${ }^{1}$ Research Organization for Information Science and Technology, 2-2-54 Naka-Meguro, Meguro-ku, Tokyo 153-0061, Japan \\ ${ }^{2}$ Center for Nanotube and Nanostructured Composites, Sungkyunkwan University, Suwon, 440-746, Korea \\ ${ }^{3}$ Department of Physics and Astronomy, Michigan State University, East Lansing, Michigan 48824-2320, USA \\ ${ }^{4}$ School of Physics, Seoul National University, Seoul 151-742, Korea \\ ${ }^{5}$ NanoCarbon Research Institute Limited, Chosei-mura, Chosei-gun, Chiba 2994395, Japan
}

(Received 8 July 2003; published 5 December 2003)

\begin{abstract}
We apply the $a b$ initio spin density functional theory to study magnetism in all-carbon nanostructures. We find that particular systems, which are related to schwarzite and contain no undercoordinated carbon atoms, carry a net magnetic moment in the ground state. We postulate that, in this and other nonalternant aromatic systems with negative Gaussian curvature, unpaired spins can be introduced by sterically protected carbon radicals.
\end{abstract}

DOI: 10.1103/PhysRevLett.91.237204

The discovery of new nanostructured carbon phases, including fullerenes [1] and nanotubes [2], has opened a new era in materials science. Minute changes in the spatial arrangement of carbon atoms can profoundly alter the electronic properties of these systems from a semiconductor to a metal or superconductor [3-6]. One could expect that structural rearrangements might also significantly change the magnetic properties of all-carbon allotropes from their known diamagnetic behavior. Only recently, a weakly magnetic all-carbon structure has been reported, formed under high-pressure and hightemperature conditions, consisting of rhombohedrally polymerized $\mathrm{C}_{60}$ molecules [7]. Other experimental observations suggest the occurrence of ferromagnetism in a semiconducting nanostructured carbon foam with a low mass density [8]. As we show in the following, the unexpected magnetic behavior of all-carbon systems can be quantitatively interpreted using spin-polarized $a b$ initio calculations. Our results suggest that unpaired spins are introduced by sterically protected carbon radicals, which are immobilized in the nonalternant aromatic system of $s p^{2}$ bonded carbon with negative Gaussian curvature. This new mechanism to generate unpaired spins in semiconductors may find a useful application in the emerging field of spintronics.

In this Letter, we investigate the electronic and magnetic properties of an $s p^{2}$ bonded all-carbon nanostructure, consisting of a nanotube junction that is structurally related to schwarzite [9-12]. This system, depicted in Fig. 1(a), is a possible building block of the recently synthesized nanostructured carbon foam $[8,13]$. Our most striking finding is that of a magnetic ground state. We find the origin of magnetism in this system to be different from previous reports [14,15], which related the spin polarization at a graphite edge to the presence of undercoordinated carbon atoms. Our calculations suggest that unpaired spins may also be introduced by carbon radicals, not only by undercoordinated carbon atoms. In the structure we propose, the radicals are sterically pro-
PACS numbers: 75.75.+a, 61.48.+c, 73.22.-f, 81.05.Tp

tected in a nonalternant aromatic system of carbons with negative Gaussian curvature.

Our electronic and magnetic structure calculations are based on the "tetrapod" geometry shown in Fig. 1(a). The core, depicted in Fig. 1(b), consists of the warped $s p^{2}$ bonded building block of the "plumber's nightmare" structure [10]. It is formed by a network of hexagons and heptagons only, and connected to four $(9,0)$ nanotube extremities. Depending on the degree of hydrogen passivation, the exposed nanotube edges are terminated by $s p^{2}$ or $s p^{3}$ carbon atoms [16]. In this type of

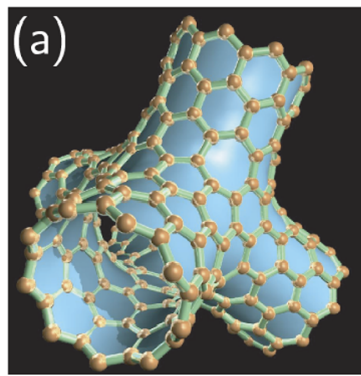

(b)
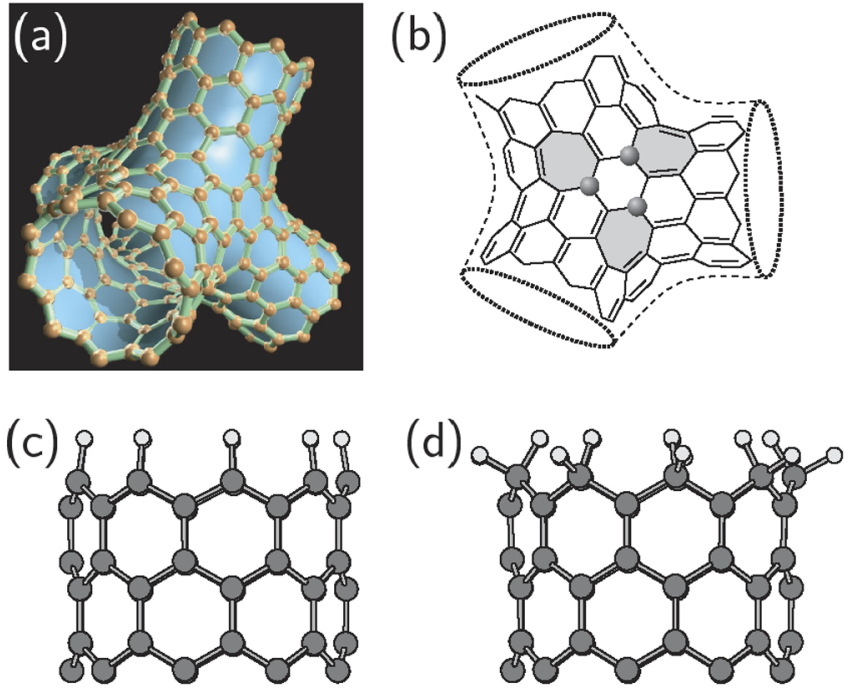

FIG. 1 (color online). (a) The structure of a carbon tetrapod, the building block of schwarzite. The four extremities consist of $(9,0)$ nanotubes with a zigzag edge. (b) Introduction of trivalent carbon radicals, emphasized by the gray spheres, in the aromatic system of the otherwise tetravalent carbon atoms in the tetrapod core. The radicals are associated with heptagons, indicated by gray shading. The structure of (c) an $s p^{2}-$ and (d) an $s p^{3}$-terminated $(9,0)$ nanotube, with the smaller spheres indicating the hydrogen atoms. 
schwarzite-related structure, tubular segments of zero Gaussian curvature coexist with saddlelike regions of negative Gaussian curvature. We expect that a range of schwarzitelike building blocks similar to ours could be cross-linked to form a foam structure with a very low mass density, and carry a magnetic moment.

We calculate the electronic structure of different tetrapods using the density functional theory within the local spin density approximation (LSDA), employing the Ceperley-Alder exchange-correlation functional for the local spin density [17,18] and ab initio pseudopotentials $[19,20]$. To facilitate the numerical treatment of very large aggregates containing hundreds of atoms within LSDA, we make use of our recently developed approach based on an atom-centered numerical basis set [21], proven to describe correctly similar carbon systems [22]. We use Pulay corrections [23] to the Hellman-Feynman forces during structure optimization.

To discriminate between the effect of the $s p^{2}$ core region and the edge region in our magnetic structure calculations, we have considered hydrogen terminated $\mathrm{C}_{264}$ and $\mathrm{C}_{336}$ clusters with the same core structure, but a different length of the nanotube extremities. We assume that the edge of these extremities may be terminated in three different ways. Besides the bare, unterminated edge, we consider an $s p^{2}$ termination by one hydrogen atom per edge atom, shown in Fig. 1(c). In the $s p^{3}$ terminated structure shown in Fig. 1(d), each carbon edge atom is connected to two hydrogen atoms. The interface between the $s p^{3}$ edge atoms and the $s p^{2}$ atoms of the nanotube is analogous to that found in diamond-graphite hybrid structures [24]. In the following, we disregard structures with a bare edge, since they are likely to be passivated fast under experimental conditions due to their high reactivity.

The electronic density of states for the $s p^{2}$ terminated tetrapod is presented in Fig. 2(a). The projection of the total density of states onto the 192 carbon atoms in the core region, including 12 heptagon rings, is shown by the solid line, and that of the 72 atoms at the edges by the dashed line. We focus on a $1 \mathrm{eV}$ wide energy window around the Fermi level, which determines the magnetic behavior, since electrons with higher binding energies are all paired. Artificial level broadening was used only in the figure, for the sake of visual clarity; no broadening has been applied during the self-consistent calculations. The density of states indicates the presence of 12 unpaired spins in this $s p^{2}$-terminated geometry. The decomposition of the density of states suggests that 8 of the 12 spins are strongly localized at the zigzag edges, with two unpaired spins at each edge.

The presence of the remaining four unpaired spin states, which are unrelated to the edge states, is independent of the length of the nanotube extremities. A detailed analysis revealed that the electronic states associated with the majority spin are not localized, but rather spread out across the entire cluster, as shown in Fig. 3(a).
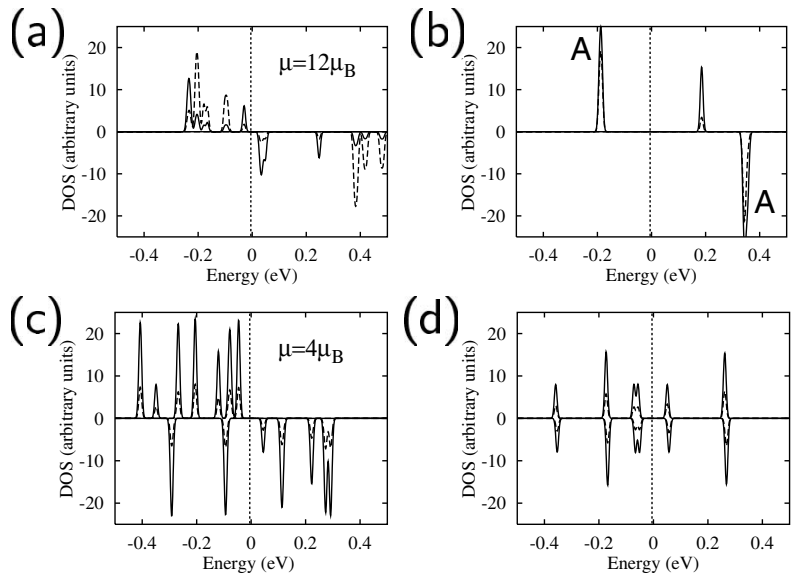

FIG. 2. Spin-resolved electronic densities of states of carbon nanostructures related to Fig. 1, with the majority spins represented in the upper panels and the minority spins in the lower panels. (a) Density of states of the $\mathrm{C}_{264}$ tetrapod of Fig. 1(a). The solid line represents the projection of the density of states onto the 192 core carbon atoms. The dashed line represents that of the 72 carbon atoms at the zigzag edges. (b) Electronic density of states of a 126-atom segment of an $s p^{2}$ terminated $(9,0)$ nanotube, shown in Fig. 1(c). The solid line represents the density of states of all the carbon atoms, and the dashed line its projection onto the 36 carbon atoms at the zigzag edge. The states denoted by " $A$ " are doubly degenerate and localized at the edge. The counterparts of (a) and (b) for $s p^{3}$ terminated structures are shown in (c) for the $\mathrm{C}_{264}$ tetrapod and in (d) for the $(9,0)$ nanotube segment. The discrete level spectrum has been convoluted with a Gaussian with a full width at half maximum of $0.012 \mathrm{eV}$. The Fermi level lies at $E=0$.

We found that the exchange splitting of the four states decreases with increasing nanotube length. This can be understood in terms of the exchange interaction between two electrons, which occupy the same region. The origin of the exchange splitting is the Coulomb repulsion between these electrons, which decreases as the region grows.

To clarify the nature of the unpaired electrons near the tetrapod edges, we performed an independent calculation for the $(9,0)$ nanotube with the same edge geometry. Similar to our results for the tetrapod in Fig. 2(a), the spin-resolved density of states of the nanotube, shown in Fig. 2(b), indicates the presence of two unpaired spins per zigzag edge. Character analysis suggests that the states in the narrow energy window used are mainly carbon based, with only a negligible contribution from hydrogen. We show the total density of states of all carbon atoms by the solid line, and that of the carbon edge atoms by the dashed line in Fig. 2(b). The spin-polarized electrons occupy doubly degenerate $\pi^{*}$ states at each edge, labeled by $\mathrm{A}$. We find one of the edge state wave functions, displayed in Fig. 3(b), to be symmetric and the other one antisymmetric with respect to a mirror plane of the nanotube. The exchange splitting of about $0.5 \mathrm{eV}$, separating the spin-up from the spin-down edge states, is 


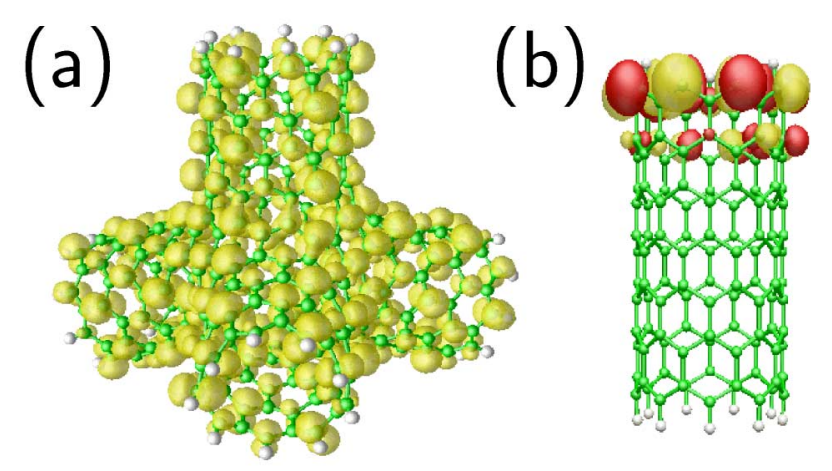

FIG. 3 (color online). (a) Charge density associated with the four unpaired spins, which are unrelated to the edge states in an $s p^{2}$ terminated tetrapod. (b) The wave function of a spinpolarized edge state in an $s p^{2}$ terminated $(9,0)$ nanotube. We use color shading to represent the phase of the wave function.

comparable to that found by ab initio plane-wave calculations for doped C-BN composite nanotubes [25].

To study the effect of edge termination on the net magnetic moment of the tetrapod, we compared our above electronic structure calculations for $s p^{2}$ terminated structures to those showing $s p^{3}$ termination. In Fig. 2(c) we present the electronic density of states for the $s p^{3}$ terminated tetrapod. To model an $s p^{3}$ termination, we attached two hydrogen atoms instead of one to each carbon edge atom, as shown in Fig. 1(d). As we noted earlier, hydrogen-induced states within this bonding geometry occur far away from the Fermi level, in analogy to the $\sigma$ states of $s p^{3}$-bonded carbon, and do not interfere with carbon-related states within the narrow energy window in Fig. 2(c). Also, the localized $\pi$ states, which we found at the edge of the $s p^{2}$ terminated structure, vanish in the $s p^{3}$ bonding geometry. In this case, we do not expect any unpaired spins associated with the $s p^{3}$ edge atoms. The projection of the density of states onto the inner 192 carbon atoms of the tetrapod is represented by the solid line, and that of the 72 edge atoms by the dashed line in Fig. 2(c). In the $s p^{3}$ terminated tetrapod, we find four unpaired spins, similar to the $s p^{2}$ terminated tetrapod, where we identified four unpaired spins, which were unrelated to the edge states.

To confirm the absence of spin polarization at the $s p^{3}$ terminated edges, we show in Fig. 2(d) the density of states of an $s p^{3}$ terminated $(9,0)$ nanotube, as a counterpart to results shown in Fig. 2(b). Our spin-resolved density of states confirms that the $s p^{3}$ edge should not be spin polarized, in stark contrast to our results for the $s p^{2}$ terminated edge. These results agree with those reported for $s p^{2}$ and $s p^{3}$ terminated graphene strips [16].

Our findings raise several intriguing questions. The first important question is, what distinguishes the magnetic structure, shown in Fig. 1(a), from other carbon structures, which are nonmagnetic, or systems, where magnetism arises due to undercoordinated edge atoms? Equally important is to find the reason for the occurrence of the spin polarization and its apparent robustness with respect to selected structural changes. To answer these questions, we calculated the magnetic ground state of numerous other $s p^{2}$ bonded carbon structures, including tetrapods terminated by $\mathrm{C}_{60}$-derived caps, fullerenes in the size range from $\mathrm{C}_{20}$ to $\mathrm{C}_{240}$, nanotube $Y$ junctions, and schwarzite-related structures with negative Gaussian curvature, containing octagons.

In our calculations, we found the number of unpaired spins not to be affected by changing size and boundary shape of the tetrapod, but to depend sensitively on the bonding topology. Setting aside the role of unsaturated graphitic edges in magnetism, which has been discussed before [14-16], we found the presence of higher polygons, introducing a negative Gaussian curvature, to be a necessary condition for a magnetic ground state. To investigate a possible conjecture that negative curvature is also a sufficient condition to establish spin polarization, we calculated the electronic ground state of a tetrapod, where all four extremities were terminated by $\mathrm{C}_{60}$-derived caps. Our finding of an energy gap of $0.34 \mathrm{eV}$ between the highest occupied and the lowest unoccupied state in this structure, which excludes the possibility of a Fermi instability, proved this conjecture incorrect.

The only viable explanation for the occurrence of magnetism in an all-carbon structure is the introduction and stabilization of carbon radicals. The occurrence of radicals, which can introduce an unpaired spin, is directly related to the possibility of pairing all valence electrons in covalent bonds. No radicals should be expected to occur if bonds in the system can be assigned in a way that would keep all carbon atoms tetravalent. In a graphitic structure, each tetravalent carbon atom is connected to three neighbors by one double and two single bonds. In such a case, all electrons should be paired and the structure should be diamagnetic. This is the case in $\mathrm{C}_{60}$ and other fullerenes, where all carbon atoms are kept tetravalent by associating single bonds with the edges of pentagons. Also in a planar graphene sheet or an infinite nanotube, all carbon atoms are equivalent and tetravalent.

Failure to find a global bonding scheme, where each carbon atom is associated with one double and two single bonds, leads to the introduction of radicals due to a frustration in the electronic system. As we expand on below, this is the case in the tetrapod structure of Fig. 1(a), where 12 trivalent carbon radicals are introduced as a consequence of inserting 12 heptagons in the otherwise alternant aromatic system of the graphitic honeycomb lattice. These trivalent carbons, emphasized in Fig. 1(b) by the gray spheres, are the source of spin polarization. In the structure discussed here, these radicals are sterically protected within the system of "single" and "double" bonds imposed by the tetrapod topology and occur in groups of three.

This conclusion can be reached by analyzing the bonding scheme in the $s p^{3}$ terminated tetrapod. The system of 
single and double bonds, shown in Fig. 1(b), follows the bonding at the edge of the terminating nanotube. Since two valence electrons of the terminating carbon atoms are paired with those of hydrogen, all bonds along the zigzag edge must be single bonds. To keep carbon atoms at the edge tetravalent, adjacent bonds in the axial direction must be double bonds, as shown in Fig. 1(b). Following this scheme into the core region of the structure necessarily leads to the above mentioned frustration, which leaves several sites, namely, the trivalent carbon radicals with a lone electron, connected to their neighbors by only three single bonds.

The lone electrons need not be localized at the radical sites, but rather gain energy by delocalizing across the entire structure, as shown in Fig. 3(a). We find the radicals to form groups of three in the tetrapods. Assuming that two of the three spins are paired, each group of three radicals should contribute one unpaired spin to the structure. With four groups of radicals per tetrapod, we would expect four unpaired spins in total, in agreement with our spin density functional calculation.

Stabilization of carbon radicals by steric protection has been known since the synthesis of triphenylmethyl in 1900 by Gomberg [26]. Radicals such as perinaphthenyl (or phenalenyl) have even been investigated as potential molecular conductors by Haddon [27]. Whereas sterically unshielded carbon-based radicals have been reported recently in organic systems [28], our study is the first report to our knowledge that identifies carbon radicals in an undoped all-carbon structure with no apparent exposed edges. We should emphasize that the magnetism found in this study is caused by the presence of sterically protected radicals within nanometer-sized conducting segments of $s p^{2}$ carbon. It could also occur in other nanostructured solids, which may be different from the tetrapod structure discussed here. We believe this to be a general phenomenon, the discovery of which may strongly influence the emerging field of spintronics.

In summary, we studied several $s p^{2}$-bonded carbon nanostructures and identified a system of unpaired electrons that is distinct from the spin-polarized states at the zigzag edge or dangling-bond states. Our spin density functional calculations for the tetrapod motif, which contains negatively curved graphitic surfaces, indicate the presence of four unpaired spins in the electronic ground state. This magnetic behavior originates in the presence of trivalent carbon radicals, which are introduced and sterically stabilized within the aromatic system of the otherwise tetravalent carbon atoms in the core of the tetrapod structure. We expect a similar type of magnetism to occur in other graphitic structures with a negative Gaussian curvature.

D. T. acknowledges partial support by NSF DMR0103587, by the Korean IMT-2000 "Molecular Logic
Devices," and by the BK21 program. N.P. and J. I. were supported by the Center for Nanotubes and Nanostructured Composites. The authors acknowledge useful discussions with Andrei Rode and thank Stuart Ramsden for assistance with scientific visualization.

*Corresponding author.

Electronic address: tomanek@pa.msu.edu

[1] H.W. Kroto et al., Nature (London) 318, 162 (1985).

[2] S. Iijima, Nature (London) 354, 56 (1991).

[3] J.W. Mintmire, B. I. Dunlap, and C. T. White, Phys. Rev. Lett. 68, 631 (1992).

[4] R. Saito et al., Appl. Phys. Lett. 60, 2204 (1992).

[5] N. Hamada, S. Sawada, and A. Oshiyama, Phys. Rev. Lett. 68, 1579 (1992).

[6] M. Schluter et al., Phys. Rev. Lett. 68, 526 (1992).

[7] T. L. Makarova et al., Nature (London) 413, 716 (2001).

[8] Andrei Rode et al. (unpublished).

[9] A. L. Mackay and H. Terrones, Nature (London) 352, 762 (1991).

[10] D. Vanderbilt and J. Tersoff, Phys. Rev. Lett. 68, 511 (1992).

[11] L. A. Bursill and L. N. Bourgeois, Mod. Phys. Lett. B 9, 1461 (1995).

[12] S. T. Hyde and M. O'Keeffe, Philos. Trans. R. Soc. London A 354, 1999 (1996).

[13] A.V. Rode, E. G. Gamaly, and B. Luther-Davies, Appl. Phys. A 70, 135 (2000); A.V. Rode et al., Appl. Phys. A 69, S755 (1999).

[14] M. Fujita et al., J. Phys. Soc. Jpn. 65, 1920 (1996); K. Wakabayashi, M. Sigrist, and M. Fujita, J. Phys. Soc. Jpn. 67, 2089 (1998).

[15] K. Nakada, M. Fujita, G. Dresselhaus, and M.S. Dresselhaus, Phys. Rev. B 54, 17954 (1996).

[16] K. Kusakabe and M. Maruyama, Phys. Rev. B 67, 092406 (2003).

[17] D. M. Ceperley and B. J. Alder, Phys. Rev. Lett. 45, 566 (1980).

[18] J. P. Perdew and A. Zunger, Phys. Rev. B 23, 5048 (1981).

[19] N. Troullier and J. L. Martins, Phys. Rev. B 43, 1993 (1991).

[20] L. Kleinman and D. M. Bylander, Phys. Rev. Lett. 48, 1425 (1982).

[21] O. F. Sankey and D. J. Niklewski, Phys. Rev. B 40, 3979 (1989).

[22] N. Park et al., Phys. Rev. B 65, 12 1405(R) (2002).

[23] M. Scheffler, J. P. Vigneron, and G. B. Bachelet, Phys. Rev. B 31, 6541 (1985).

[24] A. T. Balaban, D. J. Klein, and C. A. Folden, Chem. Phys. Lett. 217, 266 (1994).

[25] J. Choi et al., Phys. Rev. B 67, 125421 (2003).

[26] M. Gomberg, J. Am. Chem. Soc. 22, 757 (1900).

[27] R. C. Haddon, Nature (London) 256, 394 (1975).

[28] M. E. Itkis et al., Science 296, 1443 (2002). 